\title{
Peculiarities in optical response of hybrid-barrier GaSb/InAs/AISb resonant tunneling diode structure
}

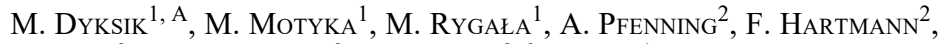 \\ R. WeiH ${ }^{2}$, L. WORSCHECH ${ }^{2}$, S. HöFLING ${ }^{2,3}$, G. SęK ${ }^{1}$ \\ ${ }^{1}$ Laboratory for Optical Spectroscopy of Nanostructures, Department of Experimental Physics, \\ Wrocław University of Science and Technology, Wybrzeże Wyspiańskiego 27, Wrocław 50-370, Poland \\ ${ }^{2}$ Technische Physik and Wilhelm Conrad Röntgen Research Center for Complex Material Systems, \\ Physikalisches Institut, Universität Würzburg, Am Hubland, D-97074, Würzburg, Germany \\ ${ }^{3}$ SUPA, School of Physics and Astronomy, University of St. Andrews, \\ St. Andrews, KY16 9SS, United Kingdom \\ ${ }^{\text {AC }}$ urrently at: Laboratoire National des Champs Magnétiques Intenses, \\ LNCMI-EMFL, CNRS UPR3228, Univ. Grenoble Alpes, Univ. Toulouse, Univ. \\ Toulouse 3, INSA-T, Grenoble and Toulouse, France
}

\begin{abstract}
We present comprehensive investigation of the optical properties of hybrid-barrier GaSb-based resonant tunneling structures, containing a bulk-like GaInAsSb absorption layer and two asymmetric type II GaSb/InAs/AlSb quantum wells. Methods of optical spectroscopy by means of Fourier-transformed photoluminescence and photoreflectance are employed to probe optical transitions in this complex multilayer system. Based on the comparison between the absorption-like and emission-like spectra (also in function of temperature) confronted with band structure calculations four main transitions could be resolved and identified. For one of them, there has been observed unusually strong linear polarization dependence never reported in structures of that kind. It has been interpreted as related to a transition at the $\mathrm{GaSb} / \mathrm{GaInAsSb}$ interface, for which various scenarios causing the polarization selectivity are discussed.
\end{abstract}

\section{Introduction}

Hybrid sensing platforms are considered as the next generation sensors for smart technology and thus strongly anticipated. Optical sensors operating in the spectral region of mid-infrared (MIR) are of particular interest due to the strongest molecular fingerprints of many gaseous substances, providing information about the environmental pollution, presence of hazardous or toxic gasses or health of an individual through the analysis of exhaled air [1]. Miniaturization and on-chip integration of methane [2] acetylene $[\underline{3}]$ or formaldehyde $[\underline{4}, \underline{5}]$ sensors presented to-date have led to units' cost reduction, thus strongly triggering the interest in the mid-infrared sensing technology 
among industries. There are already proposed solutions based on dual laser comb spectrometers [6] which, if based on semiconductor lasers, offer both, relatively easy integration and a perfect platform to perform real-time measurements. In that context as the devices of choice in the MIR, there are considered quantum cascade lasers (QCLs) [7] or interband cascade lasers (ICLs) [ $\underline{8}, \underline{9}]$, and quantum cascade detectors [10] employing different operation schemes [11]. However, they still require development and further technological and processing efforts to reach the on-chip integration.

As an emerging solution there have been demonstrated resonant tunneling diode photodetectors (RTD-PDs) [12] as a fabrication and cost-effective alternative to cascaded detectors. GaSb-based RTD-PDs exhibit spectral response in the MIR [13-15], possess much simpler layer structure and, due to its intrinsic properties, enable high current multiplication factors [16]. Thus, RTD-PD together, for instance with GaSb-based ICLs or superluminescent diodes [17], are promising candidates for realization of an on-chip low-cost gas spectrometer.

In the current work, we present a detailed analysis of the optical response and the related electronic properties of a resonant tunneling diode sample, based on a hybrid $\mathrm{GaSb} / \mathrm{InAs} / \mathrm{AlSb}$ double barrier structure (DBS), in conjunction with a bulk-like GaInAsSb absorption layer all grown on a GaSb substrate, for application in the MIR RTD-PD. We employed the Fourier-transformed temperature-dependent and polarization-resolved photoreflectance and photoluminescence measurements to probe optical transitions involving bound states confined within the DBS as well as connected with bulk absorption layer and its interface with the neighboring GaSb layer.

\section{Experiment}

The layer sequence of the molecular beam epitaxy grown structure under investigation together with the schematic band edge diagram is presented in Fig. 1a. On a p-type GaSb substrate, a $300 \mathrm{~nm}$ thick $2 \times 10^{18} \mathrm{~cm}^{-3}$ p-doped $\mathrm{GaSb}$ bottom contact layer is deposited, followed by two $30 \mathrm{~nm}$ thick GaSb-based buffer layers, $2 \times 10^{17} \mathrm{~cm}^{-3} \mathrm{p}$-doped and undoped, respectively. On top of it, there is a hybrid DBS and the following $600 \mathrm{~nm}$ thick GaInAsSb absorption layer (yellow color range in Fig. 1a) is deposited which serves as a main absorption medium of a RTD-based photodetector to get its sensitivity in the MIR [1]]. The composition of the quaternary absorption layer is adjusted to be lattice-matched to the GaSb substrate and its room temperature band gap is about $0.45 \mathrm{eV}$ [14]. The top contact layer is made of $295 \mathrm{~nm}$ thick $3 \times 10^{18} \mathrm{~cm}^{-3}$ p-doped GaSb. The entire structure is terminated with a $10 \mathrm{~nm}$ thick InAs. Figure $1 \mathbf{b}$ presents the layer sequence and the band diagram of the DBS part consisting of two asymmetric $\mathrm{GaSb} / \mathrm{InAs} / \mathrm{AlSb}$ type II quantum wells (QWs). The combination of InAs and AlSb is utilized to increase the effective barrier's height and width within the valence band, while providing an additional confinement potential for electrons in the InAs layer. 


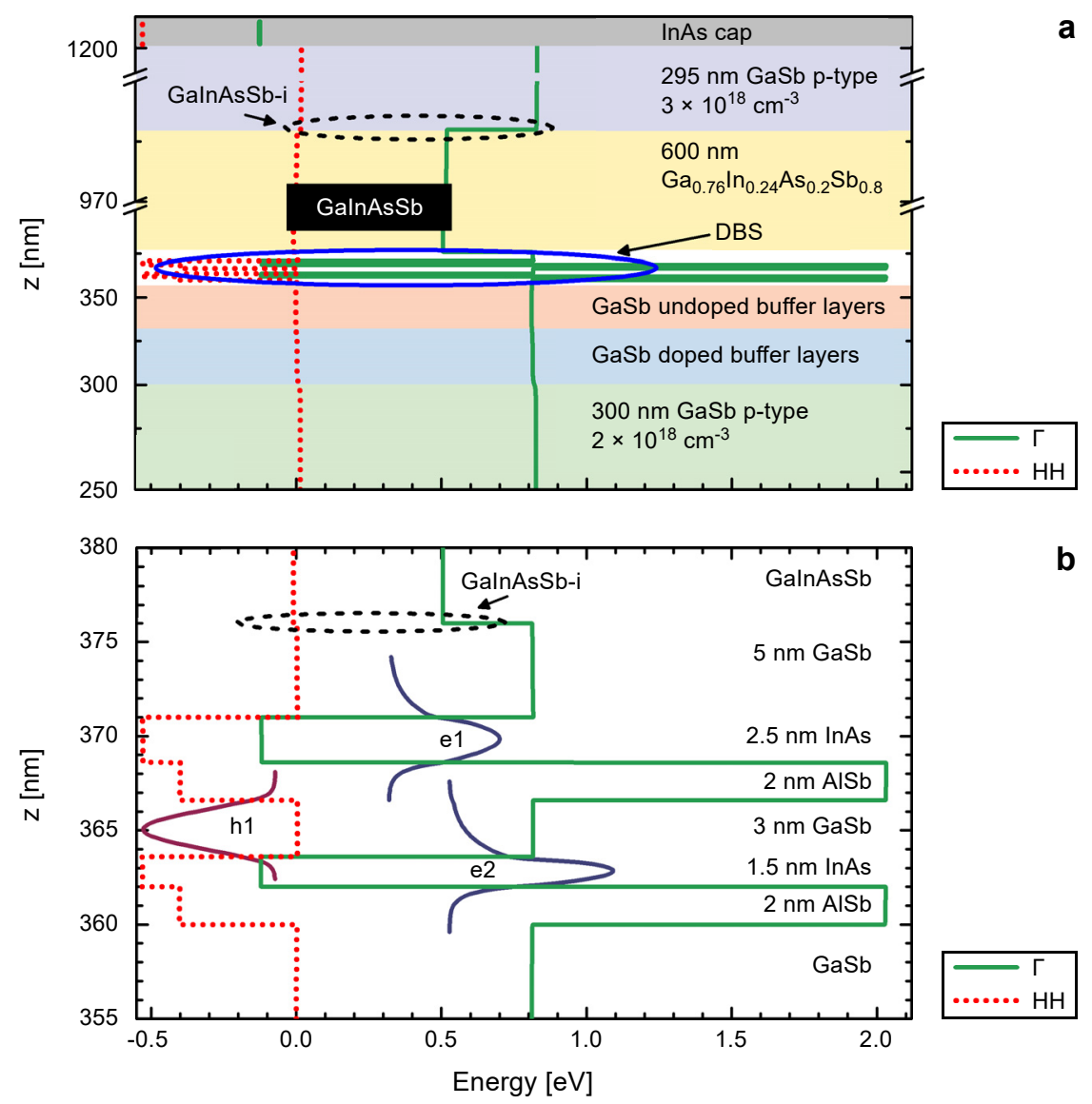

Fig. 1. The layers sequence of the investigated resonant tunneling structure together with the conduction and heavy hole band edges (a). The band alignment of the DBS consisting of two asymmetric type II $\mathrm{GaSb} / \mathrm{InAs} / \mathrm{AlSb} \mathrm{QWs}$. The probability densities of three bound states are presented (b).

Optical characterization was realized employing the Fourier-transform infrared spectrometer Bruker Vertex 80v. InSb photodetector was used in order to cover the spectral region of 1.7-5.5 $\mu \mathrm{m}$. The photoluminescence (PL) and photoreflectance (PR) measurements were performed in a step-scan mode with phase sensitive detection made by a lock-in amplifier. The PL measurements were performed in a backscattering geometry, whereas the PR measurements were performed at almost normal incidence. The $660 \mathrm{~nm} 70 \mathrm{~mW}$ laser pump beam was mechanically chopped at a reference frequency of $320 \mathrm{~Hz}$. For temperature-dependent studies, a liquid-helium continuous-flow cryostat was used with the sample mounted on a cold finger. For polarization-resolved studies the Wire Grid KRS-5 polarizer was used. The plane of polarization was determined by the polarizer's transmission axis. In order to eliminate the influence of ex- 
perimental setup, the polarization response of our system was beforehand calibrated with a tungsten lamp.

\section{Results and discussion}

The band structure properties were calculated in the framework of 8-band $\mathbf{k} \cdot \mathbf{p}$ model with the nextnano software [19]. The simulations were performed on a $0.1 \mathrm{~nm}$ grid with material parameters taken after Ref. [20]. Figure 1b presents the band alignment of the DBS with the respective QWs. For each QW the probability density of the lowest-lying state is presented, i.e. the e 1 electron state confined mostly within the $2.5 \mathrm{~nm}$ thick InAs QW, the e2 electron state confined within the $1.5 \mathrm{~nm}$ thick InAs QW and the h1 hole state confined in the $3 \mathrm{~nm}$ thick GaSb QW. The dashed lines circles in both panels of Fig. 1 indicate the location of the GaInAsSb/GaSb interface, which surprisingly also contributes to the optical response (see the discussion below). The calculated transition energies between the confined states in the DBS part, denoted as e.g. e1h1 for the transition energy between the el electron state and h1 hole state, are collected in the Table together with the transition energy for the bulk GaInAsSb absorption layer.

$\mathrm{T}$ a b 1 e. Calculated and measured transition energies at $10 \mathrm{~K}$.

\begin{tabular}{lllll}
\hline & $\mathrm{e} 1 \mathrm{~h} 1[\mathrm{meV}]$ & GaInAsSb-i $[\mathrm{meV}]$ & GaInAsSb $[\mathrm{meV}]$ & $\mathrm{e} 2 \mathrm{~h} 1[\mathrm{meV}]$ \\
\hline Calculated & 388 & - & 516 & 605 \\
PL & 386 & 468 & - & - \\
PR & 397 & 473 & 517 & 627 \\
\hline
\end{tabular}

Figure 2a presents the evolution of PR spectra with the sample's temperature. At low energy side we observed the e $1 \mathrm{~h} 1$ transition, followed by a transition we marked GaInAsSb-i, and further the signal originating from the GaInAsSb bulk absorption layer (agreeing with the expected low temperature band gap for this quaternary compound $[14,19])$. The asymmetry in the widths of the QWs building up the DBS leads to the formation of a second bound electron state $\mathrm{e} 2$ which is confined mainly on the side of the $1.5 \mathrm{~nm}$ thick InAs quantum well (see Fig. 1b). The spectral feature detected in PR at $627 \mathrm{meV}$ at the lowest temperature corresponds to e $2 \mathrm{~h} 1$ optical transition in this double QW system (its calculated value is $605 \mathrm{meV}$ - see the Table).

The expected red shifts of the PR resonances with temperature are presented as superimposed on the colored temperature-dependent PL emission map in Fig. $2 \mathbf{b}$ to allow a direct comparison. A very good agreement can be seen in these spectral ranges where both the PL and the PR signals could be detected simultaneously. In particular, the comparison could not be made for the transition at $0.627 \mathrm{eV}$ seen in low-temperature PR, because it is absent in photoluminescence. It is due to the reabsorption of this radiation in the thick quaternary layer having lower energy gap and located above the DBS. It is worth underlying here that the thin InAs does not contribute to the optical response, apparently. As it could in principle overlap spectrally with the fundamental optical transition in the DBS, we have verified that by a set of independent PL measurements 
a

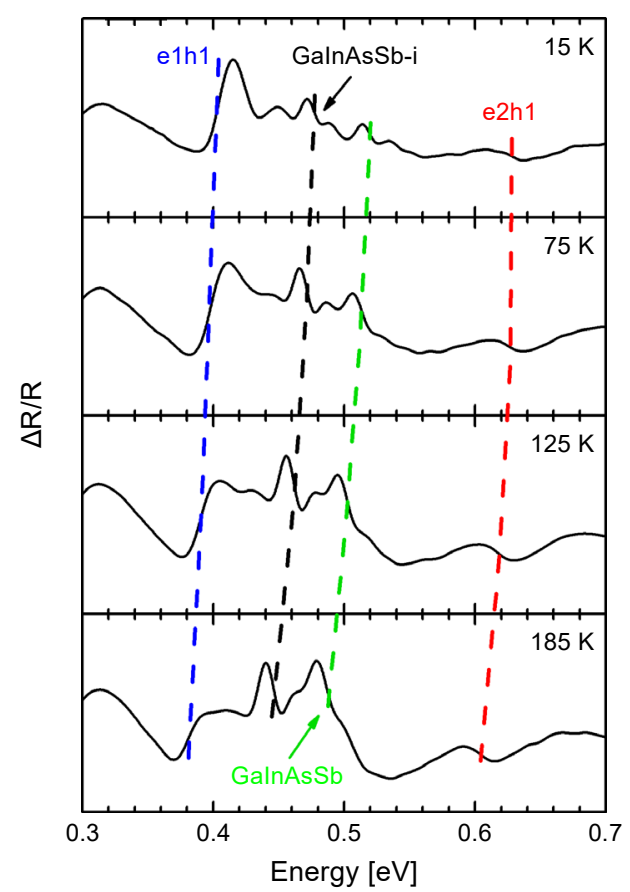

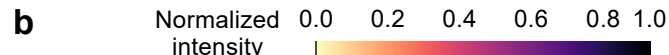

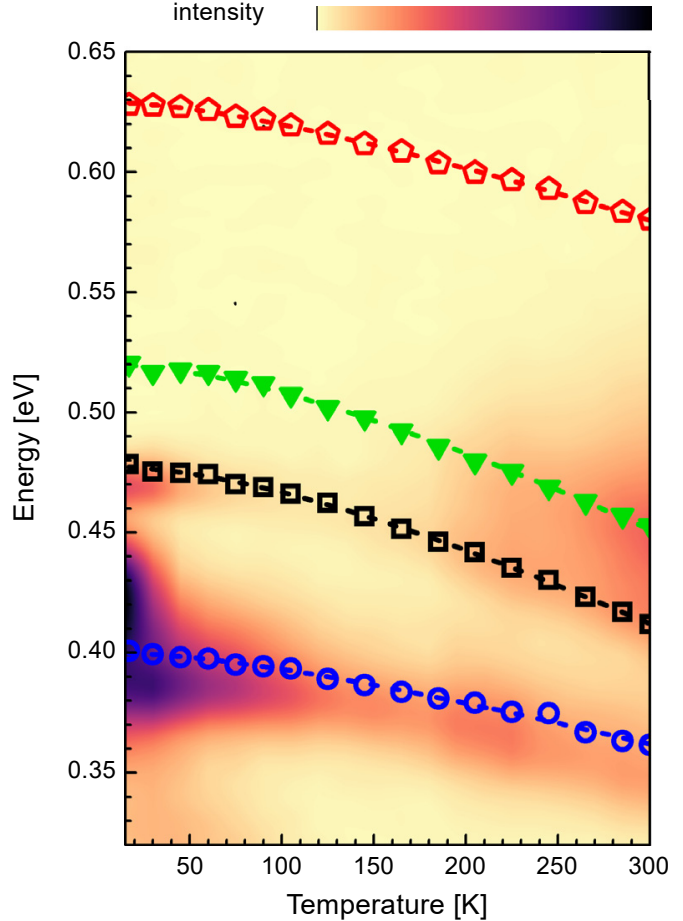

Fig. 2. Temperature-dependent PR results. Dashed lines are guides to the eye and indicate the transition energies (a). PL emission map with superimposed transition energies extracted from PR data (b).

(not shown here) on test samples with or without a similar type II InAs/GaInAsSb QW, and with or without InAs cap. The signal below $400 \mathrm{meV}$ appears only when the QW is present in the structure and no PL signal could be detected in that range for a sample with the cap and without a QW. The latter should be understandable, as this thin layer is strongly affected by the surface states which can trap the photogenerated carriers efficiently, making it mostly depleted from at least one type of carriers, which further prevents any significant band-gap-related emission.

The effective reduction of the transition energy $E$ is analyzed by means of $\alpha$ coefficient in the commonly used Varshni equation $E=E(0)-\left(\alpha T^{2}\right) /(T+\beta)$. Both the DBS-related optical transitions, e1h1 and e2h1, exhibit similar temperature dependence with $\alpha$ coefficients of $0.21 \mathrm{meV} / \mathrm{K}$ and $0.26 \mathrm{meV} / \mathrm{K}$, respectively. In the type II structure they cannot be easily related to a band gap of one of the constituting materials, but the thermal shift seems to be mostly driven by the electron states confined in the InAs layers - in agreement with even the simple intuition as these are more susceptible to changes in the confinement potential (as e.g. caused by temperature). The respective $\alpha$ coefficient for InAs bulk band gap is approximately $0.27 \mathrm{meV} / \mathrm{K}$ [20]. In contrast, the GaInAsSb-i and GaInAsSb transition energies respond much stronger to the temperature variation, with $\alpha$ coefficient of about $0.43 \mathrm{meV} / \mathrm{K}$ for both of them, being close 
to a previously reported value of $0.38 \mathrm{meV} / \mathrm{K}$ for the GaInAsSb bulk of a similar molar composition [21]. Similar values of the $\alpha$ coefficients for the former and the latter transitions' pairs indicate a common origin of both the pairs of these transitions, i.e. related to the QW and the absorption layer, respectively. It is worth underlining that the calculated electronic band structure does not exhibit any transition with energy corresponding to the GaInAsSb-i singularity, hence its origin must be different, but still related to the absorption layer (as mentioned above). Therefore it has been tentatively assigned as most probably connected with the interface between GaInAsSb and GaSb.

Figure 3 shows the polarization-resolved PL (panel a) and PR (panel b) spectra, for which we used laboratory coordinates to assign the angles (set only approximately with respect to vertical and horizontal directions), however, their supposed relations with the crystallographic directions of the sample are shown in the inset. The PL spectrum measured at a polarization angle of $0^{\circ}$ (solid line), defined by a vertically oriented

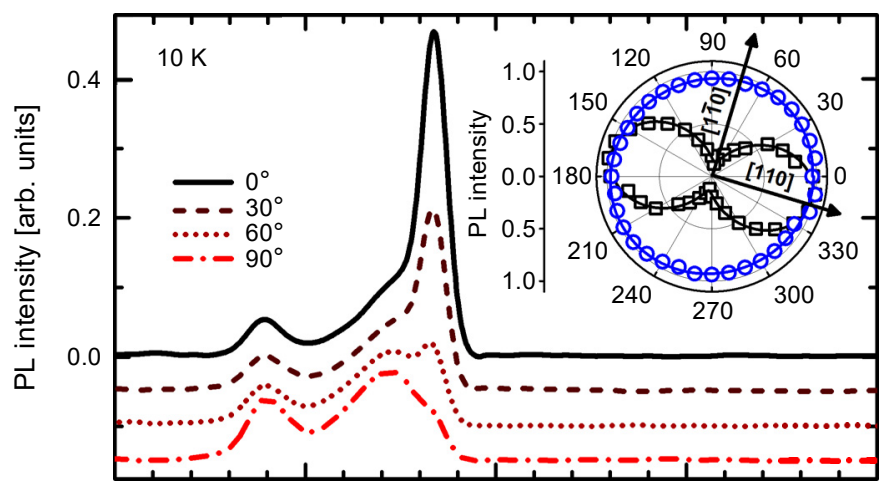

a

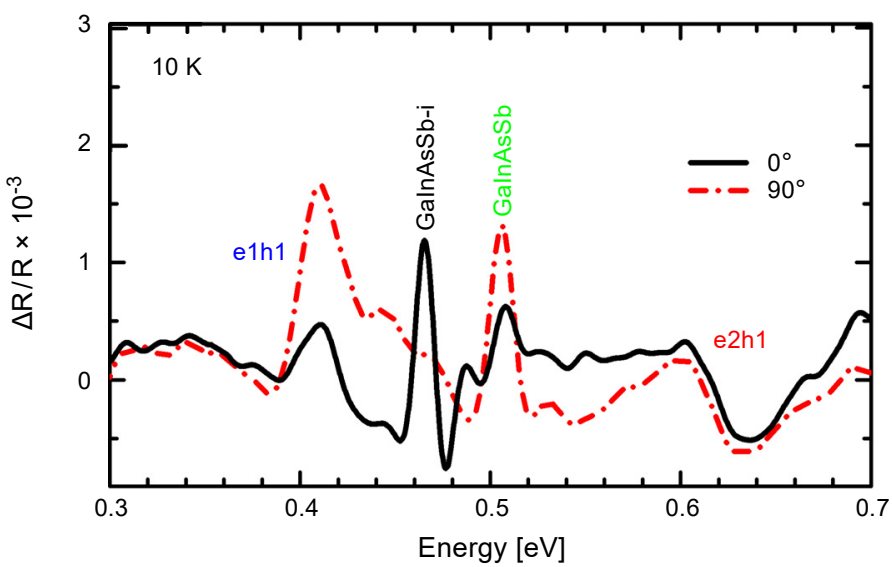

b

Fig. 3. PL (a) and PR (b) polarization resolved studies. The inset to (a) presents the PL peak intensity of elh1 (circle) and GaInAsSb-i (square) transitions as the function of polarization angle; the estimated position of the crystallographic directions [110] and [1-10] with respect to the laboratory coordinates is shown by the solid arrows. 
polarizer's transmission axis is dominated by the GaInAsSb-i transition. At the low energy side, the elh1 transition is visible. The low-temperature PR spectra measured at the same polarization angle possess, as can be expected for absorption-like spectra, two additional singularities with respect to PL data, GaInAsSb and e2h1, in agreement with the map in Fig. 2b. The red dashed-dotted line in both panels stands for the spectra measured for orthogonal orientation of the polarizer's transmission axis. One can notice a substantial reduction of the GaInAsSb-i PL peak intensity while the polarizer is rotated. On the contrary, all the other transitions present no significant polarization dependence, which comes directly from the polarization selection rules for interband optical transitions, with the chosen light propagation. The inset to Fig. 3a shows the angular dependence of the normalized PL peak intensity for the GaInAsSb-i transition (squares). The data is fitted well by Malus's law, $I(\theta)=I_{\min }+I_{\max } \cos ^{2}\left(\theta-\theta_{0}\right)$ where $\theta$ is the polarization angle and $I_{\min }$ and $I_{\max }$ are the minimum and maximum intensities. The degree of linear polarization, defined as $\left(I_{\max }-I_{\min }\right) /\left(I_{\max }+I_{\min }\right)$, is equal to 0.86 , indicating a strongly linearly polarized emission. Such a strongly polarized optical response is also visible in the PR spectra, i.e. in absorption. Both these results indicate that one deals with a transition for which a clear polarization-dependent selection rules take place for this experimental geometry. Linear polarization dependence could occur for transitions at the type II GaSb/GaInAsSb interface, formed by GaInAsSb absorption layer and the top p-type GaSb contact layer (see Fig. 1a) and/or the last (from the bottom of the structure) GaSb layer of the DBS (see Fig. 1b).

In type II zinc blende structures grown along the [001] crystallographic direction, which is the case here, the [110] and [1-10] directions are nonequivalent due to asymmetry in the bonds' alignment in the interface plane. For two compound materials with different anions and cations (which is partly present also at the interface of $\mathrm{GaSb}$ and $\mathrm{GaInAsSb}$ ), the interface consists of two nonstandard planes containing anions of one material and cations of another material, and vice versa, which means the existence of clearly oriented chemical bonds. They contribute to an asymmetry of the p-like orbitals of the valence band states at such an interface. Therefore, if one deals with a case where the carriers are localized in the vicinity of such interface and their wave functions "feel" the mentioned bonds' asymmetry, it will in turn translate into anisotropy of the optical transitions at such a type-II interface in two perpendicular linear polarizations along [110] and [1-10] directions. On a type II interface the spatially indirect transitions arise due to the electron-hole wavefunctions overlap within the region adjacent to the interface. As a result, the transition oscillator strength is strongly correlated with an anisotropic orientation of the interface chemical bonds [22]. The chemical bonds oriented in one particular direction brake the in-plane symmetry in this region, resulting in a strongly polarized emission/absorption from a nominally in-plane isotropic structure.

There can be a couple of ways or reasons for the carrier localization at the interface. One of the possible options already previously considered for similar type II systems [23] are defects. However, they usually generate relatively low densities of states and hence optical transitions involving them are easier to be observed in the emission-like ex- 
periments and are rather hardly detectable in the absorption-like spectra. Therefore, we exclude such a scenario in our case, as we observe a strong feature related to this transition also in the PR spectra.

Such a linear polarization of analyzed radiation in a backscattering geometry has been also observed both experimentally [24] and elaborated theoretically for type II interfaces of different material systems [25] and attributed there to the formation of the so called Tamm-like states at such interface. These are intrinsic states propagating parallel to the interface, but which exhibit evanescent decay perpendicular to the interface (similarly as for the Tamm surface states located energetically within the band gap).

Eventually, another possibility yet is creation of triangular potential wells at the interface (see for instance Ref. [26]), especially if layers' doping occurs as in the investigated RTD-PD structures. It has been shown in Ref. [27] (and references therein) that the exact character of the optical transition depends on the level of doping: for higher doping it takes place between electrons confined in a triangular well on the GaInAsSb side, and holes in the bulk of the same layer, whereas for moderate and lower doping it will predominantly occur between electrons localized in the narrower gap material and holes in similar triangular well on the side of GaSb. Only the latter is expected to give significant polarization anisotropy being a type II spatially indirect transition (tunneling-assisted transition in fact) with holes confined on the opposite side than the electrons. Therefore, such optical transition we actually consider as the most probable explanation of the observed in-plane polarization in our PL and PR data [료].

The current experimental data cannot exclude unequivocally any of the scenarios, as it would require decent theoretical modelling (e.g. atomistic calculations), being beyond the scope of this communication (and constituting a base for an independent report). However, these results provide for the first time an evidence for strongly polarization -dependent optical transition in such GaSb-based structures, which can have crucial consequences not only for the performance of RTD PDs themselves, but also for any other photonic devices employing doped $\mathrm{GaInAsSb} / \mathrm{GaSb}$ interface.

\section{Conclusion}

In conclusion, we have performed a comprehensive study of the optical and electronic band structure properties of a resonant tunneling diode sample designed for the mid-infrared spectral range (of 2.5-3 $\mu \mathrm{m}$ ) and featuring the type II GaSb/InAs/AlSb quantum wells with a bulk-like GaInAsSb absorption layer. Fourier-transformed photoluminescence and photoreflectance revealed optical transitions involving confined states in the QWs as well as band-to-band transition within the absorption layer. Linearly polarized photoluminescence and photoreflectance signals were observed and attributed to the localization of carriers at the GaSb/GaInAsSb heterojunction. Although the exact nature of this transition could not be defined without sophisticated theoretical modelling, existence of such type II optically active interface states in the RTD detector structure is of crucial practical importance, as they will affect the performance of the detection (by e.g. the carrier trapping) or make it polarization-sensitive. Therefore, we hope that 
our observation will stimulate further work on this phenomena, to reveal the detailed character of the interface-related and polarization-dependent transitions in the MIR, or technological efforts to reduce the interface states' and minimize their importance by optimizing the growth conditions or by post-growth processing (like annealing).

Acknowledgements - This work has been supported by grant No. 2016/21/N/ST7/02790 of the National Science Centre in Poland, and by the Polish National Agency for Academic Exchange. The authors are grateful for financial support from the State of Bavaria, the German Ministry of Education and Research (BMBF) via the national project HIRT (Grant No. FKZ 13XP5003B). M.D. acknowledges the financial support from the Foundation for Polish Science (FNP) within the START fellowship.

\section{References}

[1] GHorbani R., SchmidT F.M., ICL-based TDLAS sensor for real-time breath gas analysis of carbon monoxide isotopes, Optics Express 25(11), 2017, pp. 12743-12752, DOI: 10.1364/OE.25.012743.

[2] Dong L., Li C., SAnchez N.P., Gluszek A.K., Griffin R.J., TitTel F.K., Compact CH4 sensor system based on a continuous-wave, low power consumption, room temperature interband cascade laser, Applied Physics Letters 108(1), 2016, article 011106, DOI: 10.1063/1.4939452.

[3] Kluczynski P., Jahjah M., Nähle L., Axner O., Belahsene S., Fischer M., Koeth J., Rouillard Y., Westberg J., Vicet A., LundQvist S., Detection of acetylene impurities in ethylene and polyethylene manufacturing processes using tunable diode laser spectroscopy in the 3- $\mu$ m range, Applied Physics B 105, 2011, pp. 427-434, DOI: 10.1007/s00340-011-4645-6.

[4] Lundqvist S., Kluczynski P., Weir R., von Eduinger M., Nähle L., Fischer M., Bauer A., Höfling S., Kоетн J., Sensing of formaldehyde using a distributed feedback interband cascade laser emitting around 3493 nm, Applied Optics 51(25), 2012, pp. 6009-6013, DOI: 10.1364/AO.51.006009.

[5] Dong L., Yu Y., Li C., So S., Tittel F.K., Ppb-level formaldehyde detection using a CW room-temperature interband cascade laser and a miniature dense pattern multipass gas cell, Optics Express 23(15), 2015, pp. 19821-19830, DOI: 10.1364/OE.23.019821.

[6] Villares G., Hugi A., Blaser S., Faist J., Dual-comb spectroscopy based on quantum-cascade-laser frequency combs, Nature Communications 5, 2014, article 5192, DOI: 10.1038/ncomms6192.

[7] Faist J., Capasso F., Sivco D.L., Sirtori C., Hutchinson A.L., Cho A.Y., Quantum cascade laser, Science 264(5158), 1994, pp. 553-556, DOI: 10.1126/science.264.5158.553.

[8] Meyer J.R., Hoffman C.A., Bartoli F.J., Ram-Mohan L.R., Type-II quantum-well lasers for the mid-wavelength infrared, Applied Physics Letters 67(6), 1995, pp. 757-759, DOI: 10.1063/1.115216.

[9] YANG R.Q., Infrared laser based on intersubband transitions in quantum wells, Superlattices and Microstructures 17(1), 1995, pp. 77-83, DOI: 10.1006/spmi.1995.1017.

[10] Li J.V., Yang R.Q., Hill C.J., Chuang S.L., Interband cascade detectors with room temperature photovoltaic operation, Applied Physics Letters 86(10), 2005, article 101102, DOI: 10.1063/ 1.1875758 .

[11] Harrer A., Szedlak R., Schwarz B., Moser H., Zederbauer T., MacFarland D., Detz H., Andrews A.M., SChrenk W., Lendl B., Strasser G., Mid-infrared surface transmitting and detecting quantum cascade device for gas-sensing, Scientific Reports 6, 2016, article 21795, DOI: 10.1038 / srep21795.

[12] Pfenning A., Hartmann F., Langer F., Kamp M., Höfling S., Worschech L., Sensitivity of resonant tunneling diode photodetectors, Nanotechnology 27(35), 2016, article 355202, DOI: 10.1088 / 0957-4484/27/35/355202.

[13] Rothmayr F., Pfenning A., Kistner C., Koeth J., Knebl G., Schade A., Krueger S., Worschech L., HaRTMANN F., HöFLING S., Mid-infrared GaSb-based resonant tunneling diode photodetectors for gas sensing applications, Applied Physics Letters 112(16), 2018, article 161107, DOI: 10.1063/1.5025531. 
[14] Pfenning A., Hartmann F., Weit R., Emmerling M., Worschech L., Höfling S., P-type doped AlAsSb/GaSb resonant tunneling diode photodetector for the mid-infrared spectral region, Advanced Optical Materials 6(24), 2018, article 1800972, DOI: 10.1002/adom.201800972.

[15] Nie B., Huang J., Zhao C., Huang W., Zhang Y., Cao Y., Ma W., InAs/GaSb superlattice resonant tunneling diode photodetector with InAs/AlSb double barrier structure, Applied Physics Letters 114(5), 2019, article 053509, DOI: 10.1063/1.5079923.

[16] Pfenning A., Hartmann F., Rebello Sousa Dias M., Langer F., Kamp M., Castelano L.K., Lopez -Richard V., Marques G.E., HöFling S., Worschech L., Photocurrent-voltage relation of resonant tunneling diode photodetectors, Applied Physics Letters 107(8), 2015, article 081104, DOI: 10.1063 / 1.4929424.

[17] Zia N., Viheriälä J., Koivusalo E., Virtanen H., Aho A., Suomalainen S., Guina M., GaSb superluminescent diodes with broadband emission at $2.55 \mu \mathrm{m}$, Applied Physics Letters 112(5), 2018, article 051106, DOI: 10.1063/1.5015974.

[18] Hartmann F., Pfenning A., Knebl G., Wein R., Bader A., Emmerling M., Kamp M., Höfling S., WORSCHECH L., In Antimonide-based resonant tunneling photodetectors for mid infrared wavelength light detection, Proceedings of SPIE 10403, 2017, article 1040306, DOI: 10.1117/12.2274917.

[19] Birner S., Zibold T., Andlauer T., Kubis T., Sabathil M., Trellakis A., Vogl P., Nextnano: general purpose 3-D simulations, IEEE Transactions on Electron Devices 54(9), 2007, pp. 2137-2142, DOI: 10.1109/TED.2007.902871.

[20] Vurgaftman I., Meyer J.R., Ram-Mohan L.R., Band parameters for III-V compound semiconductors and their alloys, Journal of Applied Physics 89(11), 2001, pp. 5815-5875, DOI: 10.1063/1.1368156.

[21] Wen'GANG B., Aizhen L., Band gap energy and its temperature dependence of GaInAsSb quaternary alloy grown by molecular beam epitaxy, Chinese Physics Letters 9(1), 1992, pp. 53-56, DOI: $10.1088 /$ 0256-307X/9/1/015.

[22] Platonov A.V., Kochereshio V.P., Ivchenko E.L., Mikhailov G.V., Yakovlev D.R., Keim M., Ossau W., WaAg A., Landwehr G., Giant electro-optical anisotropy in type-II heterostructures, Physical Review Letters 83(17), 1999, pp. 3546-3549, DOI: 10.1103/PhysRevLett.83.3546.

[23] Shen J., Goronkin H., Dow J.D., Ren S.Y., Tamm states and donors at InAs/AlSb interfaces, Journal of Applied Physics 77(4), 1995, pp. 1576-1581, DOI: 10.1063/1.358910.

[24] Wu S., Chen Y., Yu J., Gao H., Jiang C., Huang J., Zhang Y., Wei Y., Ma W., In-plane optical anisotropy of InAs/GaSb superlattices with alternate interfaces, Nanoscale Research Letters 8, 2013, article 298, DOI: 10.1186/1556-276X-8-298.

[25] Ivchenko E.L., NeSTOKLON M.O., Lateral optical anisotropy of type-II interfaces in the tight-binding approach, Physical Review B 70(23), 2004, article 235332, DOI: 10.1103/PhysRevB.70.235332.

[26] Donetsky D., Anikeev S., Belenky G., Luryi S., Wang C.A., Nichols G., Reduction of interfacial recombination in GaInAsSb/GaSb double heterostructures, Applied Physics Letters 81(25), 2002, pp. 4769-4771, DOI: 10.1063/1.1530743.

[27] Mikhailova M.P., Moiseev K.D., YaKovlev Y.P., Interface-induced optical and transport phenomena in type II broken-gap single heterojunctions, Semiconductor Science and Technology 19(10), 2004, pp. R109-R128, DOI: 10.1088/0268-1242/19/10/R03.

[28] Mikhailova M.P., Titkov A.N., Type II heterojunctions in the GaInAsSb/GaSb system, Semiconductor Science and Technology 9(7), 1994, pp. 1279-1295, DOI: $\underline{10.1088 / 0268-1242 / 9 / 7 / 001}$.

Received February 3, 2020 in revised form July 18, 2020 